\section{P361 PREVALENCE OF BACTERIAL VAGINOSIS AMONG WOMEN ATTENDING SEXUAL HEALTH CLINICS IN NEW YORK CITY, 2017-2018}

${ }^{1}$ Alissa Davis*, ${ }^{2}$ Preeti Pathela, ${ }^{2}$ Addie Crawley, ${ }^{3} J u l i a$ Schillinger. ${ }^{1}$ Columbia University, School of Social Work, New York, USA; ${ }^{2}$ New York City Department of Health and Mental Hygiene, Bureau of Sexually Transmitted Infections, New York City, USA; ${ }^{3}$ Centers for Disease Control and Prevention, New York City, USA

\subsection{6/sextrans-2019-sti.464}

Background Bacterial vaginosis (BV) is a common condition among women and is associated with HIV/STI acquisition and adverse pregnancy outcomes. We describe the prevalence of BV diagnoses and repeat BV diagnoses among women attending New York City (NYC) public sexual health clinics (SHC) and examine demographic characteristics associated with BV.

Methods Using electronic medical record data for patients who were assigned female sex at birth ("women"), we identified BV diagnoses made during clinician visits at NYC SHC during January 2017-April 2018, and recurrent BV during a six-month follow-up period through October 2018. BV diagnosis was defined as a physician diagnosis of BV with a vaginal $\mathrm{pH} \geq 4.5$, and positive whiff-amine test. We used chisquare tests to compare characteristics of women with and without a BV diagnosis.

Results Of 13,153 women with $\geq 1$ visits to the SHC, $34 \%$ $(4,449)$ were diagnosed with BV. There were significant differences in BV prevalence by race/ethnicity: 39.5\% (95\% CI: [38.7-40.3\%]) of non-Hispanic black women versus $29.6 \%$ ([28.3-30.9\%]) Hispanic, 21.2\% ([19.1-23.3\%]) non-Hispanic white, and 19.7\% ([16.5-22.9\%]) non-Hispanic Asian women. Women of HIV-negative/unknown HIV status had higher BV prevalence than HIV-positive women $(33.9 \%$ vs $23.4 \%$, $\mathrm{p}=0.02)$. Women reporting sex with both men and women had higher BV prevalence (42.0\%, 95\% CI: [37.5-46.5\%]) than women reporting sex with men only (34.5\%, [34.2$34.8 \%])$ or women reporting sex with women only $(28.1 \%$, [22.2-34.0\%]). Among women with BV, 4,404 (99\%) received treatment and 1588 (36.1\%) of those had another clinician visit within 6 months. Among returning women, 282 (17.8\%) were given another BV diagnosis within 3 months, and 601 $(37.8 \%)$ within 6 months.

Conclusion One-third of women attending NYC SHC were diagnosed with BV, and recurrent BV was common. Additional research is needed to identify effective therapies to reduce the high prevalence and recurrence rates of BV.

Disclosure No significant relationships.

\section{P363 COMBINED ORAL AND TOPICAL ANTIBIOTIC THERAPY FOR MALE PARTNERS OF WOMEN WITH BACTERIAL VAGINOSIS: A PILOT STUDY}

\begin{abstract}
${ }^{1}$ Erica Plummer*, ${ }^{1}$ Lenka Vodstrcil, ${ }^{2}$ Christopher Fairley, ${ }^{3}$ Jennifer Danielewski, ${ }^{3}$ Gerald Murray, ${ }^{2}$ Michelle Doyle, ${ }^{2}$ Glenda Fehler, ${ }^{2}$ Marti Kaiser, ${ }^{2}$ Colette Mcguinness, ${ }^{3}$ Suzanne Garland, ${ }^{4}$ Dieter Bulach, ${ }^{2}$ Eric Chow, ${ }^{5}$ Jane Hocking, ${ }^{2}$ Catriona Bradshaw. ${ }^{1}$ Monash University, Central Clinical School, Carlton, Australia; ${ }^{2}$ Alfred Health, Melbourne Sexual Health Centre, Carlton, Australia; ${ }^{3}$ The Royal Women's Hospital, Centre for Women's Infectious Disease Research, Parkville, Australia; ${ }^{4}$ Melbourne Bioinformatics, The University of Melbourne, Carlton, Australia; ${ }^{5}$ University of Melbourne, Melbourne School of Population and Global Health, Parkville, Australia
\end{abstract}

10.1136/sextrans-2019-sti.465
Background More than 50\% of women experience recurrence of bacterial vaginosis (BV) within 3-6 months following firstline antibiotics. Increasing evidence suggests that reinfection from an untreated sexual partner contributes to BV-recurrence. We conducted a pilot study of combined oral and topical antibiotic treatment of male partners of women being treated for BV.

Methods Women attending Melbourne Sexual Health Centre with symptomatic BV ( $\geq 3$ Amsel's criteria and Nugent Score $[N S]=4-10)$ were recruited with their regular male partner (RSP). Women received oral metronidazole $400 \mathrm{mg}$ twice daily (BD; or $2 \%$ clindamycin cream nocte, if contraindicated), for 7-days. Males received oral metronidazole $400 \mathrm{mg} B D$ and $2 \%$ clindamycin cream topically to penile skin $B D$, for 7-days. Couples self-collected genital samples (vaginal swab, penile swab and male urine) and completed questionnaires for 4weeks post treatment. The proportion of women cured (NS $\leq 7)$ at 4-weeks post treatment was calculated. A subset of couples will be followed to 12 -weeks post treatment and this data will also be available for presentation. Genital samples will be analysed to understand the impact of partner treatment on the genital microbiota, and the contribution of behaviours and host factors to BV-recurrence.

Results Data from 46 couples has been analysed. Thirty-eight women $(83 \%)$ reported a past history of BV and $14(30 \%)$ reported intra-uterine device (IUD) use. Most RSPs were uncircumcised $(n=38,83 \%)$. Treatment adherence was high, with $91 \%$ taking $\geq 70 \%$ of prescribed doses. The proportion of women cured at 4-weeks was 93\% ( $n=43 / 46$, 95\%CI: $82-$ 99\%). All cases of BV-recurrence $(n=3)$ had a past history of BV and an uncircumcised RSP. Two cases had an IUD in situ and one reported condomless vaginal sex during treatment.

Conclusion Treating sexual partners of women with BV may be an effective strategy for improving BV-cure. Randomised controlled trials of partner treatment currently underway will help determine the effectiveness of this intervention.

Disclosure No significant relationships.

\section{P364 GARDNERELLA VAGINALIS CLADE DISTRIBUTION IS ASSOCIATED WITH BEHAVIOURS AND NUGENT SCORE IN WOMEN WHO HAVE SEX WITH WOMEN}

${ }^{1}$ Erica Plummer*, ${ }^{1}$ Lenka Vodstrcil, ${ }^{2}$ Gerald Murray, ${ }^{1}$ Christopher Fairley, ${ }^{2}$ Jennifer Danielewski, ${ }^{2}$ Suzanne Garland, ${ }^{1}$ Eric Chow, ${ }^{3}$ Dieter Bulach, ${ }^{4}$ Katherine Fethers, ${ }^{5}$ Jane Hocking, ${ }^{1}$ Catriona Bradshaw. ${ }^{1}$ Monash University, Central Clinical School, Carlton, Australia; 'The Royal Women's Hospital, Centre for Women's Infectious Disease Research, Parkville, Australia; ${ }^{3}$ Melbourne Bioinformatics, The University of Melbourne, Carlton, Australia; ${ }^{4}$ Alfred Health, Melbourne Sexual Health Centre, Carlton, Australia; ${ }^{5}$ University of Melbourne, Melbourne School of Population and Global Health, Parkville, Australia

10.1136/sextrans-2019-sti.466

Background Gardnerella vaginalis (GV) can be detected in women with BV and without BV. Identification of four genetically distinct GV-clades (defined using clade-specific genetic markers) led to the hypothesis that there may be both pathogenic and commensal GV-clades. We conducted a study investigating the distribution and behavioural associations of GVclades in a cohort of women-who-have-sex-with-women (WSW).

Methods Women self-collected vaginal swabs and completed behavioural questionnaires 3-monthly for 24 months or until incident BV(Nugent Score[NS]=7-10). qPCR assays were used to detect $\mathrm{GV}$ and the four GV-clades. Multinomial logistic 